\title{
The intrinsically linked future for human and Artificial Intelligence interaction
}

Anthony Miller ${ }^{*}$ (D)

${ }^{*}$ Correspondence:

a.miller12@unimail.derby. ac.uk

Department of Engineering

\& Technology, University

of Derby, Derby, England, UK

\begin{abstract}
No sleep! No sick days! No Holidays! alongside 24/7 around the clock tireless work, welcome to the world of Artificial Intelligence, how can humans match the phenomena of Al. Humanity is in the grip of an unstoppable, and exciting future with the future development of Al, humans must decide whether to ride on the coat-tails of $\mathrm{Al}$ or resist the inevitable change of the world as we know it. The real game changer will be the commercial availability of Quantum Computing. Humans must learn to work, live, learn and interact with Al or become second class citizens. Big data is the lifeblood of Al and Quantum Computing processing power will enable future computers to process incredible amounts of big data. Al is taking over the job's human do, receptionists, drivers, chefs and in the future doctors and accountants. Quantum Supremacy will soon be achieved, and Al will soon reach and far exceed the "Singularity". Will humans grasp the opportunity to develop with Al or resist? humans have the chance to develop with Al through brain computer interface $(\mathrm{BCl})$ technology. Security and regulations have to be put in place, with questions of "Who is responsible for Al security and regulations"? and "can Al be trusted as an autonomous entity" also the ethical use of Al has to be addressed, "What about the rights and ethics of Al"? The human race is on an inevitable path of Al dominance the question is "will humans and Al be friends or adversaries"?
\end{abstract}

Keywords: Quantum Computing, Artificial Intelligence, Big Data, Human and Al interaction

\section{Introduction}

Human and artificial intelligence interaction are presenting prodigious and exciting technological opportunities for mutual development even in today's current technological climate, but the real potential for mutual development is in the foreseeable future and beyond which has the potential to be mind-boggling. With the continued rapid development of not just technology but also in quantum research the potential for Artificial Intelligence to evolve at a frightening speed is within our grasp. The building blocks to achieve this phenomenal leap in technology consists of Quantum Computing, Big Data and brain computer interface (BCI), each becoming stepping stones and advancing to such a stage where once technology only thought of in science fiction will be an everyday feature of modern human and AI technology. AI and human beings bring their own unique traits with each learning from the other, humans bring attributes including experience, values and judgement which can weave together with the enormous attributes

(c) The Author(s) 2019. This article is distributed under the terms of the Creative Commons Attribution 4.0 International License (http://creativecommons.org/licenses/by/4.0/), which permits unrestricted use, distribution, and reproduction in any medium, provided you give appropriate credit to the original author(s) and the source, provide a link to the Creative Commons license, and indicate if changes were made. 
that AI can contribute. AI and human interaction have the potential to develop and push the boundaries of not just technologies on earth but also space exploration. For example, with this collaboration comes the potential for the successful development and commercial use of Nuclear Fusion to supply a clean new vast energy source. Add this together with the ability of AI to negate the need to rest, to feed or the need to breath oxygen, giving AI the ability for vast space travel while investigating possible future human exploration and even pushing the boundaries further with potential human colonisation. Vast amounts of big data and the plentiful supply of structured data are essential for the future advancement of AI, the training of learning algorithms for use in Deep Learning and Neural Networks are essential to make AI more intelligent.

The processing power of today's computers are already working in conjunction with big data continuously advancing the ability to process big data more efficiently evolving alongside the advent of parallel processing and Hadoop with both advancing at a frenetic rate which in turn is enabling AI to become even more intelligent. Imagine the progress of $\mathrm{AI}$ if the power of Quantum Computing was readily available with potential processing power a million times more powerful than today's classical computers with each manufacturer striving to reach not just Quantum Supremacy but far and beyond this revolutionary breakthrough. Humanity can harness this amazing technology with $\mathrm{AI}$ and $\mathrm{BCI}$ generating a new technology revolution, this is the future, but the future is fast approaching. With Quantum Computing bringing the ability to process big data in abundance this will in turn skyrocket AI development leading to the advancement of BCIs, with AI having the ability to not just pinpoint but to map and increase for example DNA mapping identifying genes in strength and intelligence. The possibility for super strength and super intelligent humans to match the huge advancement in AI intelligence is attainable. With the singularity certain to be surpassed in the future humans and AI will strive for Artificial General Intelligence (AGI). This potential for Humans and AI to grow and develop together is staggering but the question of Security, Regulations and Ethics alongside AI and future human $\mathrm{BCI}$ advancements highlights the need for standard security and regulations to be put in place.

With this paper, we analyse previous papers from Big Data, Artificial Intelligence, Quantum Computing, brain computer interface (BCI), AI Security, Regulations and Ethics as well as papers on the future of AI. The papers are recent publications, published between 2013 and 2018, the papers we have chosen all have relevant categories in them in relation to AI and Human Interaction, Security Regulations and Ethics and future AI alongside Big Data. Furthermore, we analyse categories of the aforementioned papers and investigate the relevant categories to be reviewed in each paper. With the categories that relate to our chosen topic of AI and Human Interaction we will intertwine and present a subset of papers from each of our researched paper categories.

The paper is organised in the following order. Firstly, in "Big Data and AI" section Big Data and Artificial Intelligence are analysed alongside their intertwined relationship focusing on the Three Vs of Volume, Variety and Veracity [1] Hadoop and the future potential for explosive technological advancement between the Big Data and AI. "Quantum Computing, Big Data and AI" section examines the potential cataclysmic growth of Big Data and AI when Quantum Computing becomes another piece of the future jigsaw puzzle for AI and Human Interaction. Contributing factors in 
"Human and AI interaction" section for AI and human interaction is the need for both $\mathrm{AI}$ and humans to learn from each other and the potential now and in the future. "Brain, computer interface (BCI)" section investigates brain computer interface (BCI) and the ways AI and Humans use each other's strengths to grow together now and in the future. AI Security, Regulations and Ethics are dissected and analysed in "AI, security, regulations and ethics" section. Finally, a conclusion to the paper is in "Conclusions and recommendations" section.

\section{Big Data and Al}

"The most successful AI applications rely heavily on big data" [2]. "Big Data is perhaps best described by IBMs three Vs, Volume, Variety and Velocity" [1]. Volume denotes huge amounts of data generated by numerous sources, including healthcare, manufacturing, communications and banking to name just a few, but two of the latest largely untapped sources due to explode in the near future are chat bots processing data from human and AI interaction and the Internet of Things gathering huge amounts of data collected from each new device attached to the network. Variety represents the numerous ways data can be used to analyse a predicament or event. Different kinds of devices generate different kinds of data both structured and unstructured, hospital equipment, web browsing and of course the IOT. "In 2011555 million websites and over 100 million blogs each containing videos, pics text and audio generated vast amounts of big data" [1] this has now grown exponentially with big data growing at an unprecedented rate. Velocity is increasing rapidly day by day, with the advancement of technology so advances the velocity of big data. "There are more than 250 million tweets a day each leading to response and discussion tweets" [1] escalating the velocity of big data enormously.

The emergence of Hadoop and has allowed us to analyse and process big data faster and more efficiently than ever before using distributed and parallel techniques, alongside the emergence of the cloud both have the capability to process vast amounts of data. "Algorithms currently have limitations which are being addressed with a machine learning process in Hadoop with capabilities such as recommendation mining, clustering and classification" [1]. "Yahoo is the largest user of Hadoop with over 500 million uses per month and billions of transactions per day" [1]. "The process of MapReduce is also used by Google for many activities including data mining and machine learning" [1]. AI and big data are intrinsically linked, the more big data that AI processers the more intelligent it becomes with the cycle becoming bigger and more advanced day by day. "A Twitter investigation of messages discovering hashtags and emotions linked to sentiment uncovering new streams of related big data" [1]. "Already half the world's stock trades are done by AI" [1], with the advancement of more sophisticated applications for the processing of unstructured big data, quality big data will be become far more readily available. With AI being used for the processing of unstructured data big data will continue to grow exponentially day by day. "AI can be used to identify and clean dirty data or use dirty data as a means of establishing context knowledge" [1]. AI is continuing to evolve and generate new avenues of big data with video analysis and visual machine learning contributing to the advancement of AI. 


\section{Quantum Computing, Big Data and AI}

Humanity has an ever growing and insatiable appetite for big data. "Classical or even today's super computers cannot keep pace with the huge increase in big data and the processing this entails" [3]. "Due to these limitations' quantum mechanics is taking the role of game changer in the race of computation" [3]. Quantum Computing is needed to analyse and process the extraordinary growth in big data now and in the future with the IOT and chatbots forecast to contribute vast amounts of new data in the future. For example, "a telco in the city of Shenzhen in China serves 101 million users producing 5 tb of big data a day" [4] this creates millions of records a day and multiplying the number of cities and countries globally classical computers cannot process this amount of big data, so Quantum Computing is the logical future progression. In classical computers we have binary bits of 0 and 1 , in quantum we have Qubits, in classical a bit can be 0 or 1 at a given time, Qubits can be 0,1 and other states at the same time, this is called Superposition with each Qubit capable of infinite states at the same time.

"If Qubits are combined, then the number of states rise exponentially with quantum computers estimated to be a million times faster than a classical computer" [3]. Quantum Supremacy (when Quantum Computing out performs classical supercomputers) is predicted to be reached in 2019 with Google predicting a 72 Qubit computer. To put in perspective a 64 Qubit quantum processor is 2 to the power 64 meaning a 64-bit classical computer with typical speed combinations of 2 billion a second would take 400 years to match the power of the quantum processing. This technology has some major hurdles to overcome to become a main stream technology, processors require temperatures to be near absolute zero $\left(-273.15{ }^{\circ} \mathrm{C}\right)$, there needs to be zero interference from the environment, also packing and combining the Qubits along with the state of Superposition needing to be attained for longer periods of time in order to perform the quantum calculations. When Quantum Computing becomes viable this will have a knock-on effect for big data and AI providing the ability to process any amount of big data generated. With big data being the lifeblood of AI, the intelligence of AI will grow to unimaginable levels.

\section{Human and $\mathrm{Al}$ interaction}

"Researchers have highlighted the need to make AI ready for human interaction" [5]. Human beings need to learn from AI and AI needs to learn from human beings. "The field of human-robot interaction uses an experimental technique called the Wizard of $\mathrm{Oz}$ where the human operator remotely controls the behaviour of AI" [5]. The Wizard of $\mathrm{Oz}$ is a technique which enables AI to provide appropriate reactions to human behaviour as well as human expectation. A Wizard of Oz Interface is already in development which in turn leads to further AI and human development. "Socially guided machine learning reframes the machine learning problem as an interaction between the human and machine" [5].

Machine intelligent quotient (MIQ) is a cloud-based system meaning it is online 24/7. This is a system where AI learns from human behaviour and humans control the direction of the AI machine. This kind of interaction enables not just AI to learn from human behaviours but also AI to learn from human AI interaction. Experiments are already under way for example in hospitals for the development of robots who act as a 
support mechanism for patients with lifelong ailments including diabetes or autism. AI has already proved that the provision of physical activity with adults is within its grasp. Chatbots for AI and human interaction are already at an advanced stage ready for worldwide use, but the main topic of controversy and debate is AI being used for military purposes and the huge issue of autonomous $\mathrm{AI}$ in warfare without human interaction making decisions for itself.

\section{Brain, computer interface (BCl)}

"Brain machine interface (BMI) allows users to control external devices by analysing their brain signals" [6]. BCIs are now used for experiments not just online but also data analysis offline by using open source toolboxes. This can offer people with severe disabilities a chance for ground breaking communication with the outside world. BMI is still at an early stage with only a small number of brain signal algorithms available, but this is an incredible opportunity for $\mathrm{AI}$ and human interaction.

"Using collaborative BCI with the target of improving human response in Visual Target Recognition is currently in development" [7]. "One can achieve higher levels of perceptual and cognitive performance by leveraging the power of multiple brains through BCI collaboration" [7].

Collaborative BCIs have the objective of improving human performance by using neural data from multiple brains in combination with advanced signal analytics meaning the more brains that are used the greater the performance with support vector machine (SVM) technology being utilised to classify the data.

AI with quantum technology can for example be used for DNA mapping with the possibility of the human genes of strength and intelligence identified meaning BCIs can be used to implement and enhance these traits for future human development.

\section{Al, security, regulations and ethics}

"We have to think what the moral issues are and how we can use Artificial Intelligence to make a better decision for both the people and society" [8]. Human beings use emotions in consideration of making decisions, this is at present missing from AI. At present human beings build the learning algorithms which AI uses to analyse data. This raises the question of not just the engineer who builds the algorithm having bias tendencies, (which they probably do not realise) but also the data that the engineer is using to build the algorithm with, what bias data does this contain? Currently there is no security or regulations governing the widespread use of big data so different cultures are certainly going to suffer from AI bias. Certain corporations have floated the idea of regulating themselves while developing AI algorithms and develop AI ethics and security at the same time. Currently there is rapid development of $\mathrm{AI}$ in the military which brings the need for not just security but also regulations, this also highlights the risk of autonomous AI making decisions on aspects of warfare without standard regulations or security. "Ethical decisions are being faced to support or oppose autonomous weapons systems" [8]. "It would be a security threat if AI with self-aware ability could not be fully controlled by humans" [2]. "Ethics is a concern with the moral behaviour of the machine which is designed and used for many purposes" [8]. AI is advancing rapidly but ethics and security are not, $\mathrm{AI}$ is involved with DNA mapping, what security, regulations and 
ethics are developers following? AI needs to make a decision regarding human casualties, how does it choose which casualty to choose the few or the many, regulations are needed in such a scenario.

"Many uncontrolled consequences might arise from AI applications if we fail to identify and prevent related threats beforehand" [2]. "In June 2015 a failed robot on an assembly line in America attacked a worker leading to the workers death" [2]. In future AI can self-evolve to a stage where human beings could potentially be put at risk, is this a risk humanity is prepared to take without adequate safeguards in place?

"The most successful AI applications rely heavily on big data" [2] this alone brings huge privacy problems.

"Big data is currently a hot research topic; however big data can contain sensitive information and must therefore be sufficiently protected as it is stored and protected" [9]. AI will evolve to such an extent that in the future there will be a possibly of self-regulation with AI regulating AI and hence becoming autonomous. "We find that security and privacy for big data based on reviewed papers is not different from security and privacy research in general" [9], this could possibly be a starting point for standard security regulations and ethics across the $\mathrm{AI}$ industry.

\section{Conclusions and recommendations}

The future is upon us! Quantum supremacy will be achieved and surpassed, AI singularity will be achieved and surpassed, Quantum Computing is the key to unlock untold possibilities bringing never before seen challenges to the human race. Big Data is the lifeblood of AI and will increase exponentially with the advancement and commercial availability of Quantum Computing to process vastly more big data than at present, this will in turn make AI far more intelligent than humans, raising the question of "how will the human race cope with no longer being the most intelligent species in the universe"? (as far as we know). Humans must embrace AI and advance together for mutual future development; this collaboration will bring the possibility of discoveries that until now have been beyond human capabilities alone.

For example, the discovery of Tachyons, these particles can travel faster than the speed of light, thus disproving Einstein's Theory of Relativity, Tachyons are able to escape the gravity of a black hole unlike light, so this opens up the possibility of investigations into what happens inside a black hole. The universe is also expanding faster than the speed of light, this is caused by dark energy, the discovery of dark energy alongside dark matter bringing the solution to the Theory of Everything would further enhance the development of human beings and AI. With these advancements who knows how many Tachyons and sub atomic particles can be discovered in the future.

AI doesn't need sleep, rest, food, or need air to breathe, these benefits working in conjunction with a clean and abundant energy source created for example from Nuclear Fusion, then deep space exploration is a distinct possibility leading to possible human colonisation of planets.

If humans do not grasp the potential AI interaction which will bring together each other's best traits, then humans will certainly be second class citizens in their own world. AI processes vast amounts of data far faster than humans can ever contemplate whereas humans bring emotional traits such as empathy, joy, and judgement, bringing the best 
of both attributes together can make the future for humans and AI far more beneficial, albeit humans will need AI far more than AI needs humans. The rising growth of earth's population combined with the huge increase in resources needed to sustain the human race are unsustainable, it is inevitable that humans will outgrow earth and will need to colonise new planets to survive, also looking into the future in billions of years terms our sun will eventually burn out and earth will cease to exist.

Another possible huge advancement would be in DNA mapping, AI could identify the genes for strength, speed and intelligence and enhance these genes making humans far superior than they are now. The identification of medical diseases and the possibly eradication of these genes giving the potential for humans to live longer which in turn greatly increase the world's population.

In future AI will have chat bots on a world-wide scale interacting with humans, solicitors, accountants and midwifes will be potential robots. This continued interaction with humans will enable future advancement and development from both sides on a huge scale. Robot midwifes in future will possibly be delivering humans with superhuman traits compared with today's humans due to DNA mapping. With brain computer interface (BCI) technology AI can identify, enhance and implant these genes making humans faster stronger and more intelligent.

The Internet of Things (IOT) will provide huge amounts of new big data with future chatbot technology available to every household bringing constant AI and human interaction. With Quantum Computing processing huge amounts of big data this in turn will enable Wizard of $\mathrm{Oz}$ and Artificial General Intelligence (AGI) technology to thrive with AI learning from human characteristics, this can be used in conjunction with $\mathrm{BCI}$ technology to advance AI and human interaction to an unprecedented level in the future.

Humans must embrace and evolve alongside AI to continue to develop as a species, the potential of $\mathrm{BCI}$ technologies to rapidly advance will bring the evolution a new breed of human and AI interaction. The commercial viability of Quantum Computing is the key to the huge advancements in AI and human interaction.

Another scenario to speak of is classical computer cryptography, with quantum processes having the ability to decipher classical computer encryption. With Quantum Computing becoming more advanced quantum cryptography will become far more secure and advanced with the completely new design of cryptography for classical computers needed due to the vulnerability the power of Quantum Computing will bring. This advancement also brings with it a new set of problems, cybersecurity with the possibility of hackers infiltrating AI. AI must be hacker proof and AI will eventually be used to provide cryptography for AI itself. This will potentially eradicate another huge problem which has been around since the age of the philosopher Aristotle, the problem of AI bias. Each individual nation has some kind of critical infrastructure this at present relies on classical computer cryptography this will need to be drastically updated when Quantum Computing becomes commercially available. With the vast computing power of Quantum Computing becoming more advanced this will eventually mean the end of classical cryptography and classical computers themselves.

Quantum Computing will enable AI to scale heights that have never before been seen, a huge increase in processing power and available big data will lead to far more intelligent AI. 
AI is developing far faster than security ethics and regulation protocols which cannot keep pace with this continued advancement. Organisations are floating the idea of security and ethics self-regulation, this must be avoided as the corporate world firstly has a responsibility to profits and shareholders with the possibility of security ethics and regulations becoming part of a balance sheet on their corporate infrastructure.

An industry recognised council is required to govern AI security regulation and ethics, one that regulates and governs the corporate world also setting a standard across the board which the corporate world has to adhere to, this has to be a prerequisite of future $\mathrm{AI}$ research and not a choice of corporate entities to be involved or not. Each government can issue licenses to companies and have the option to withdraw them if companies do not adhere to the council's standards. This must be adhered to on a world-wide scale with all nations adhering to a common set of rules for security, regulation and ethics.

With the potential of AI to evolve at such a frightening rate AGI will be achieved and surpassed bringing another potential issue of AI ethics, AI will evolve to an as yet unimaginable level of intelligence that AI will then raise the question of AI ethics and how this will be implemented in the future.

It is imperative that potential individual gene DNA mapping to name just one future $\mathrm{AI}$ and human advancement brings the need for stringent regulation it is imperative that humans stay in control of AI especially where warfare is concerned. AI cannot be allowed to self-regulate and become autonomous until further research has been carried out with Quantum Computing advancing AI to huge technological advancement, for example deep learning and a complete understanding of how AI makes decisions within hidden layers must be fully understood before even contemplating autonomous AI.

The future of the human race is extricably linked to AI and must be faced concomitantly, commercial viability of Quantum Computing will change future AI and human interaction immeasurably.

Abbreviations

Al: Artificial Intelligence; $\mathrm{BCl}$ : brain computer interface; AGl: artificial general intelligence; BMI: brain machine interface; IOT: Internet of Things; DNA: deoxyribonucleic acid.

\section{Acknowledgements}

Not applicable.

Authors' contributions

The author contributed to the research and manuscript in its entirety. All authors read and approved the final manuscript.

Funding

Not applicable.

Availability of data and materials

Not applicable.

Competing interests

The authors declare that they have no competing interests.

Received: 13 March 2019 Accepted: 6 May 2019

Published online: 13 May 2019

References

1. O'Leary DE. Artificial intelligence and big data. In: IEEE intelligent systems; 2013. p. 96-9. https://doi.org/10.1109/ mis.2013.39. 
2. Li X, Zhang T. An exploration on artificial intelligence application: From security, privacy and ethic perspective. In: IEEE 2nd international conference on cloud computing and big data analysis (ICCCBDA); 2017. p. 416-20. https:// doi.org/10.1109/icccbda.2017.7951949.

3. Singh J, Singh M. Evolution in quantum computing. In: International conference system modeling \& advancement in research trends (SMART); 2016. p. 267-70. https://doi.org/10.1109/sysmart.2016.7894533.

4. Costa C, Zeinalipour-Yazti D. Telco big data: current state \& future directions. In: 19th IEEE international conference on mobile data management (MDM); 2018. p. 11-4. https://doi.org/10.1109/mdm.2018.00016.

5. Magyar G, Vircikova M. Proposal of a cloud-based agent for social human-robot interaction that learns from the human experimenters. In: IEEE 19th international conference on intelligent engineering systems (INES); 2015. p. 107-11. https://doi.org/10.1109/ines.2015.7329648.

6. Lee MH, Fazli S, Kim KT, Lee SW. Development of an open source platform for brain-machine interface: open BMI. In: 4th international winter conference on brain-computer interface (BCl); 2016. p. 1-2. https://doi.org/10.1109/iwwbci.2016.7457440.

7. Yun K, Stoica A. Improved target recognition response using collaborative brain-computer interfaces. In: IEEE international conference on systems, man, and cybernetics (SMC); 2016. p. 002220-3. https://doi.org/10.1109/ smc.2016.7844568.

8. Kumar N, Kharkwal N, Kohli R, Choudharym S. Ethical aspects and future of artificial intelligence. In: International conference on innovation and challenges in cyber security (ICICCS-INBUSH); 2016. p. 111-4. https://doi. org/10.1109/iciccs.2016.7542339.

9. Nelso B, Olovsson T. Security and privacy for big data: a systematic literature review. In: IEEE international conference on big data (big data); 2016. p. 3693-702. https://doi.org/10.1109/bigdata.2016.7841037.

\section{Publisher's Note}

Springer Nature remains neutral with regard to jurisdictional claims in published maps and institutional affiliations.

\section{Submit your manuscript to a SpringerOpen ${ }^{\circ}$ journal and benefit from:}

- Convenient online submission

- Rigorous peer review

- Open access: articles freely available online

- High visibility within the field

- Retaining the copyright to your article

Submit your next manuscript at $\gg$ springeropen.com 